\title{
STAlz: remotely supporting the diagnosis, tracking and rehabilitation of patients with Alzheimer's
}

\author{
Hélder Moreira and Renato Oliveira \\ Associação Fraunhofer Portugal Research \\ Rua Alfredo Allen - 455/461, 4200-135, Porto \\ Email: \{helder.moreira, renato.moreira\}@ fraunhofer.pt
}

\author{
Nuno Flores \\ Department of Informatics Engineering, \\ Faculty of Engineering, University of Porto \\ Rua Dr. Roberto Frias, s/n 4200-465 Porto \\ Email: nflores@fe.up.pt
}

\begin{abstract}
As the World's population gets older, diseases directly related to aging are increasingly becoming the focus of technological effort and research. Dementia, in general, and Alzheimer's disease (AD), in particular, is one of the most common afflictions among elderly people, with studies predicting an even bigger incidence in the next decades. Due to mobility constraints, patients are being inadequately followed by their medical staff, hindering an effective delay of the disease's progression through preventive therapeutics. As such, new ways of diagnosing, tracking and rehabilitating AD patients are needed. This paper presents STAIz, a mobile device based system developed to provide caregivers and medical professionals a way to be in contact and share important information, and to provide patients with a tool that could potentiate the exercise of their cognitive functions. This system was tested with Alzheimer's disease caregivers and the initial results proved promising.
\end{abstract}

\section{INTRODUCTION}

Life expectancy has been rising in the last decades. Senior mortality rate is lower due to improvements in health and social care. People are living longer and, combined with a lowering birth rate, the average age of the population is increasing [1].

There are several diseases associated with aging, being dementia one of the most prevalent categories. Within it, Alzheimer's disease (AD) is the most common affliction, responsible for up to 60 to $80 \%$ of all the cases [2]. The disease can display symptoms such as memory loss, difficulty in carrying everyday tasks, change in mood and personality, and disorientation to time, place and people. It is a major cause of disability in the elderly, affecting the quality of life of both patient and caregiver, and burdening the public health system [3]. According to the Alzheimer's Association, it is estimated that the disease costs, annually, on average, 172 billion dollars globally [2].

Furthermore, diagnosing, monitoring and rehabilitating AD in a patient is a delicate and long process, not only because of the disease itself but because this situation accounts for three different roles: patient, caregiver and doctor. Each of these actors brings additional difficulties into the process, as there is a need to gather them in the same place, at the same time, each one having different obligations.

The patient is likely an elderly person, who the disease may have already impaired some of his cognitive functions. The need for a caregiver becomes indispensable, not only to accompany the patient, but to provide important monitoring information. Advanced age might also bring mobility issues, restraining the patient's availability for more periodic doctor appointments.

The majority of Alzheimer's caregivers have full or part time jobs. Their responsibilities at work are adversely affected by the demands of caregiving. Two thirds of working Alzheimer caregivers reported that they missed work because of their caregiving responsibilities: $14 \%$ gave up work entirely or chose early retirement; $13 \%$ cut back on their work hours or took a less demanding job; $8 \%$ turned down a promotion and $7 \%$ lost job benefits[4].

Most doctors are part of a public health care system, having on their care dozens of patients where dementia may only represent a small part of their workload [3]. Normally, an AD patient doesn't have a regular, highly frequent, appointment routine. This hinders the physician's ability to gather and interpret the patient's symptoms and behaviors more thoroughly. Alas, it prevents performing rehabilitation in an interval that would be meaningful and that could provide more favorable results.

The need arises to seek solutions for improving the way, treating and monitoring $\mathrm{AD}$ patients is performed. These should thrive to minimize the burden, both economical and psychological, the disease poses on all three actors.

This paper presents STAlz, a mobile device based system, aimed at improving the way that monitoring, diagnosing and rehabilitation is done in $\mathrm{AD}$ patients. It does so by providing:

- $\quad$ Frequent monitoring and evaluating metrics - A way to monitor the patient remotely through the caregiver, in smaller intervals than what is done currently $(>6$ months);

- Remote rehabilitation - A way for the patient to be able to do cognitive stimulation exercises remotely, while allowing the doctor(s) to personalize this training and analyze the patient's progress;

- Increased proximity - A way of establishing a more permanent contact with the caregiver, in two ways: (1) relieving and reassuring him, by providing answers to frequently asked questions (FAQ) and situations associated with the $\mathrm{AD}$ and (2) allowing for the caregiver to report important situations that otherwise would wait until the next doctor's appointment;

- $\quad$ Support to health care professionals - A way to support the occupational therapist in his work, providing 
a system that he/she can use when visiting the patient at home.

The remainder of the paper is organized as follows: section II will describe the context and problems that STAlz addresses, by analyzing the related work on the area; sections III and IV detail the conception and implementation behind the mobile and web components of the system, respectively; section $\mathrm{V}$ elaborates on the preliminary results of using the system and section VI concludes the paper, proposing future work directions.

\section{MONITORING PATIENTS With AlZHEIMER'S}

Alzheimer's disease is an incurable, neurodegenerative condition with symptoms such as memory loss, disorientation in time, space and people, among others. It poses as a heavy burden to the patient, the caregiver and the health care system.

Feedback gathered from interviews with medical professionals ${ }^{1}$ suggests that there are still issues with current $\mathrm{AD}$ monitoring, where the most prominent are: (1) tracking the patient evolution more frequently, by gathering patient indicators as to the stage of the disease and (2) analyze these indicators as to speed up the diagnostic process and improve therapeutics. Research conducted by the authors haven't, so far, found the existence of technological solutions that specifically aim at mitigating these issues altogether. Nevertheless, there are contributions is related areas that are deemed relevant for the presented work.

Within the domain of cognitive stimulation, cognitive rehabilitation produces significant improvements in targeted areas (such as AD), at least for a part of treated patients, therefore, alternative and innovative ways of memory rehabilitation in the AD context can be effective and useful [5] [6].

Studies have shown [7] [8] that a patient's memory can, in fact, be preserved and in some cases improved.

Memory rehabilitation results can be enhanced if provided at the time of both information acquisition (patient is trying to memorize new information) and information retrieval (patient is trying to recall memorized information). In this context, computer-assisted training supports this enhancement by providing exercises aimed at stimulating learning of new information and retrieval from semantic memory [5]. This self-improves the computer tests' performance as well as the outcome of traditional cognitive assessment tests. These indicators suggest that the rehabilitation of $\mathrm{AD}$ patients, while computer-supported, is a process that can provide good results in maintaining (and even improving) cognitive functions. However, the technological solutions found to provide this kind of rehabilitation have still show some gaps.

COGWEB [9] and Lumosity [10] are platforms that despite providing a good range of cognitive exercises, require a personal computer (PC) with an active connection to the internet. RehaCom [11], a solution widely used at hospitals and occupational centers, requires the patient to attend these facilities in order to perform the exercises, since special hardware is needed to interact with the platform.

\footnotetext{
${ }^{\mathrm{I}}$ The authors interviewed several experienced professionals, specialized in treating AD patients.
}

STAlz aims at complementing these solutions by providing similar cognitive stimulation exercises in a mobile device, either a tablet or a smartphone, without requiring the patient to travel to a designated facility. The system can be used at home, with the supervision of the caregiver. Its viability comes from the fact that, nowadays, mobile devices are more common due to its accessible price (when compared to a laptop) and the introduction of touch interactivity. These can lead to further developments in designing cognitive exercises. STAlz also removes the need for an permanent, active internet connection.

\section{STALZ SYSTEM FOR MOBILE DEVICES}

The STAlz's main goal is to provide medical professionals with relevant information about the patient's progress, while empowering the patient with a way to perform cognitive rehabilitation exercises. All in a mobile environment without the need of an internet connection.

It serves as the means that both caregiver and patient will have at their disposal to communicate with the medical professionals. Since an internet connection may not always be available, the system was designed to work offline. Synchronization with a shared central server is assumed to occur when an internet connection becomes available, at the responsibility of the caregiver.

In order to maximize the effectiveness of the application from an end-user perspective, a prototyping approach was followed. By developing rapid prototypes and performing usability tests, the authors were able tune the design and adapt the functionalities to the actual needs of the caregivers and patients. These usability tests and their results can be found in section V.

\section{A. Design concerns}

The application is targeted at both caregivers and AD patients, thus, its user interface had to be tailored to both audiences. Design aimed at being responsive, minimalist and easy to understand. A special attention had to be given to the patients, mostly elderly people with slim to none experience with smartphones and/or tablets. It was imperative that they understood what was asked of them at all times. Otherwise, the captured information would be severely compromised, failing to differentiate valid (although incorrect) answers from answers to misunderstood questions.

Accounting for accessibility issues, a high contrast design was chosen, to maximize the user ability to read and fully understand all the texts presented in the application. It was also favored big buttons and large font sizes, to make sure the users have no trouble clicking/tapping the options or reading/understanding the meaning of the texts throughout the application.

Regarding safety, the main menu was designed as a "home" screen with four main options. The idea behind this dashboard (seen in Figure 1) is that, at any given time, the user can always go back to this screen and restart the task. Its comprised of only four "big" buttons, becoming a place easy to recall, thus providing the user with a fail-safe mechanism, if they, somehow, feel lost within the application. 


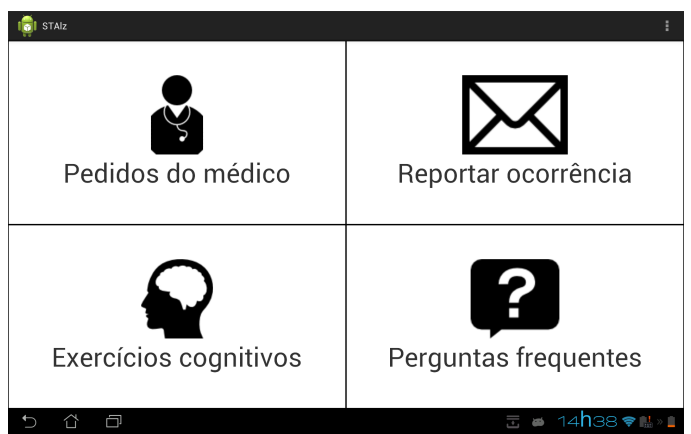

Fig. 1. Dashboard of the application with it's four main areas: to-do tasks, report occurrence, cognitive exercises and frequently asked questions

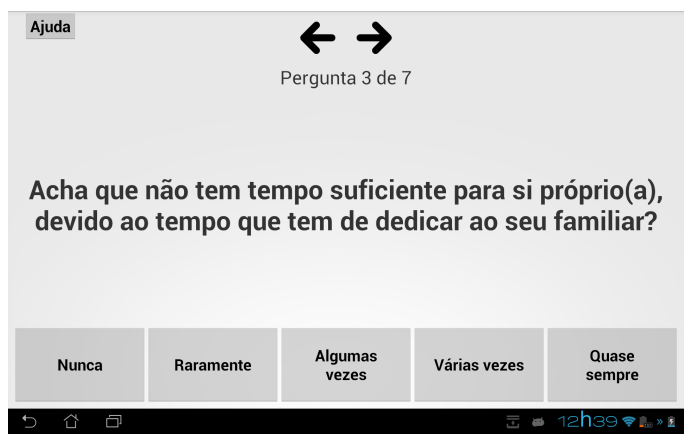

Fig. 2. Example of a question in the Zarit Burden Interview questionnaire. The minimalistic interface focuses on the question and possible answers. To accommodate situations such as reviewing answers and changing them, a navigating tool (arrows) inside the interview was also included.

\section{B. Components}

This section describes the STAlz mobile system's main components.

1) Interviews to the caregiver: Since the patient may no longer be able to answer questions truthfully, interviews like Zarit Burden Interview (ZBI) [12] (example in Figure 2), Instrumental Activities of Daily Living (IADL) [13] and the Neuropsychiatric Inventory (NPI-Q) [14] were included as system features. These interviews share one common denominator: they can be answered by the caregiver, and provide information about both caregiver and patient. By including them, it provides the medical professionals with the option to prescribe these interviews, appearing in a "to-do tasks" area of the system for the caregiver to perform. If answered and delivered at short time intervals, these interviews provide the doctor with useful diagnostic information, helping with a more adequate evaluation of the patient's condition and required changes in the course of treatment.

2) Cognitive Exercises: As suggested by the interviewed medical professionals, in order to achieve positive results with cognitive stimulation, exercises need to be performed in a 2 to 3-day interval. The challenge was then to define which candidate exercises would provide the desired stimulation and be feasible in a mobile environment (done at home, sometimes without supervision, in still early stages of the disease).

One of the main goals was to keep the subject engaged in the exercises. To motivate the patient, increasing levels of difficulty were considered, having the exercise adapting
Toque no ecrã na sequência correta

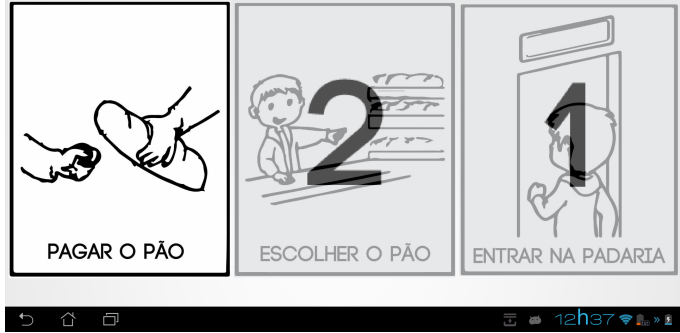

Fig. 3. IADL cognitive exercise where the patient needs to identify the correct order of the sub-actions to form an instrumental activity of daily life.

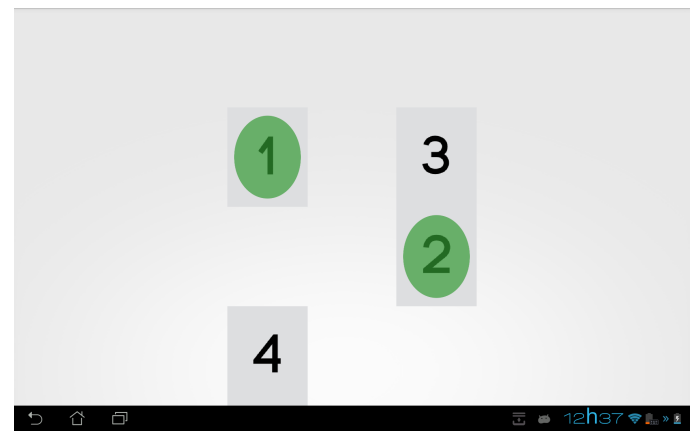

Fig. 4. Number sequence cognitive exercise - the patient needs to identify the correct sequence of numbers.

itself automatically (without visual feedback) according to the patient's progress and results.

Three cognitive pilot exercises were chosen that aim at stimulating the patient's executive functions, attention, memory and language:

- IADL - The goal in this exercise is for the patient to identify the correct order in a series of images provided. The images form a task or action associated with the instrumental activities of everyday living which are normally easier for the patient to identify (See Figure 3). The amount of images varies according to the difficulty level.

- Number sequence - This exercise asks the patient to identify the correct order of a sequence of numbers (See Figure 4). The patient is required to tap/select the numbers in the correct order. The length sequence varies according to the difficulty level.

- $\quad$ Memory - In this exercise, the patient is presented with a grid, where some cells are filled (See Figure 5). After a given time, all cells are cleared and the patient needs to recall which cells were filled (See Figure 6). Images can be used as filling. The grid size, number of images and time before the images disappear varies according to the difficulty level.

3) Occurrences: To better understand the patient's progress, it is imperative that medical professionals are aware of every relevant occurrence in the patient's behavior. An out of the ordinary agitated moment, a non-related clinical incident or a change in behavior are examples of occurrences, that despite 


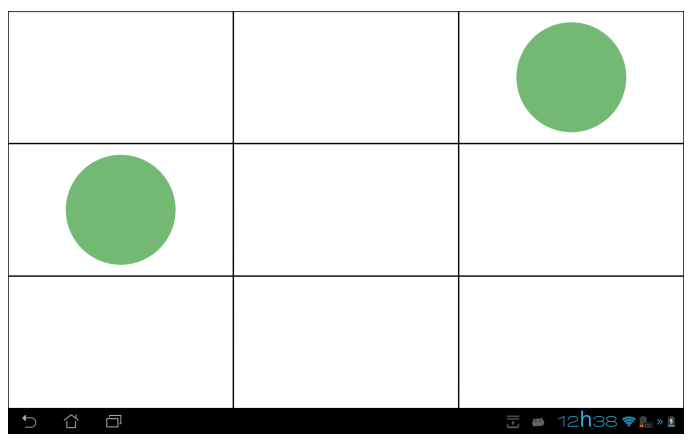

Fig. 5. Memory cognitive exercise - initial state where the positions are shown to the patient for a pre-defined number of seconds.

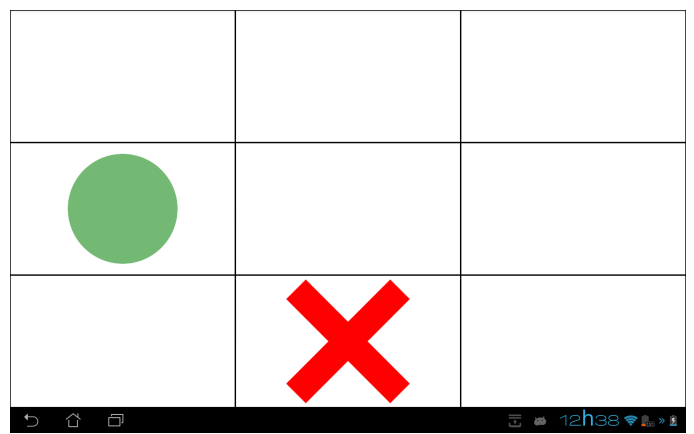

Fig. 6. Memory cognitive exercise - state where the patient is guessing the position previously shown.

relevant, are not always accurately reported. The application provides the caregiver with means to register and report these occurrences back to the medical staff. The caregiver has the option to report occurrences in both text and audio, enabling a detailed description of what happened (or is still happening) and thus alerting the medical specialists.

4) Frequently asked questions: Many caregivers are faced with the same questions when it comes to the Alzheimer's disease. How to deal with the sleep patterns? How to react to a certain behavior? How to improve the house environment to avoid accidents? and so on. Answers for these and other questions are provided by the system, where the caregiver can browse through a list of answers to "frequently asked questions", based on knowledge provided by medical specialists and professionals.

The purpose is to provide the caregiver with information that helps dealing with everyday events and also to reassure that many of the situations that occur are common and not accountable. Knowing that other people are having the same issues is, according to medical experts, one of the best ways to reassure the caregiver, allowing for comfort and confidence to ensure a good performance.

\section{STALZ SYSTEM FOR THE WEB}

The medical professionals have access to a web platform where they can access all data gathered by the system that regards their patients. This platform was designed given the inputs of medical professionals in the area. They elicited which data was most relevant for diagnostic purposes and that could be captured remotely. The platform allows for the following:
- Diagnostic metrics. When the patient is performing the cognitive exercises, data such as: where the user clicked, whether it was a correct or incorrect click, when did the click occur, level of difficulty, number of attempts, and so on, is stored for analysis. These metrics allow the doctor to ascertain of the progress of the patient's condition. Regarding interviews, it is possible to analyze each individual answer, as well as the overall score and compare it to previous ones. By doing this analysis, it is possible to establish patients' and caregivers' state and progress, determining the best course of treatment.

- Alerts. The occurrences reported by the caregiver are given special attention since they represent a probable urgent situation and therefore personal alerts (via email, for instance) can be configured by the medical staff to be aware when new situations occur.

- $\quad$ Task prescription. Besides accessing the patient's history and information, there is also the possibility to prescribe tasks for both the caregiver and the patient. These tasks can be interviews, cognitive exercises, or simply an instruction that the medical professional wishes to ask the caregiver or patient to do.

\section{INITIAL RESULTS AND ANALYSIS}

Usability tests were performed with Alzheimer's caregivers in order to evaluate the system's usability throughout the development phase. The caregivers were chosen according to three main characteristics: whether the caregiver wears glasses, has a cellphone and is familiar with a touch device. Considering these characteristics, a pool of 10 caregivers was scrutinized to try and represent the potential target users of STAlz. As seen in Table I, $50 \%$ of the subjects wear glasses, $80 \%$ of the subjects own a cellphone and $50 \%$ of them have never experienced a touch device. This allowed for us to cover various possibilities ranging from subjects with sight problems, to subjects with no previous experience with touch devices or with cellphones or technology at all.

TABLE I. LIST OF SUBJECTS AND RELEVANT CHARACTERISTICS

\begin{tabular}{|c|c|c|c|c|}
\hline Subject & Age & Glasses? & Cellphone? & $\begin{array}{c}\text { First time } \\
\text { touch device? }\end{array}$ \\
\hline 1 & 27 & No & Yes & No \\
\hline 2 & 49 & No & No & Yes \\
\hline 3 & $>50$ & No & No & Yes \\
\hline 4 & 26 & No & Yes & No \\
\hline 5 & 21 & No & Yes & No \\
\hline 6 & 50 & Yes & Yes & No \\
\hline 7 & 45 & Yes & Yes & No \\
\hline 8 & $>50$ & Yes & Yes & Yes \\
\hline 9 & 61 & Yes & Yes & Yes \\
\hline 10 & 62 & Yes & Yes & Yes \\
\hline
\end{tabular}

Each subject was asked to simulate three use cases:

- The medical professional sends a task, asking the caregiver to answer a Zarit Burden Interview. 
- The caregiver has doubts on how to deal with the patient's food habits and tries to find the answer in the application;

- $\quad$ Something unusual has happened with the patient, and the caregiver wishes to report that to the doctor.

Throughout the test, the caregiver was monitored for performance. Every misclick, click in the wrong location and, in general, wrong action by the caregiver was counted as an error. At the end of the test, the subjects were questioned on their opinion on the usefulness of the system.

Their results can be seen on Table II.

Performance - The results show that even people with no previous experience in smartphone/tablet devices were able to perform the core activities of the system, such as answering an interview, consulting a FAQ and reporting an occurrence to the attending physician. It is also possible to state that the number of errors progressively decreased throughout the tests, indicating that the design decisions taken (increasing font sizes, spacing between buttons, etc.) improved the system's overall usability. These initial results suggest that with a clean and minimalist interface, even people with no experience whatsoever with touch devices can perform tasks associated with monitoring and tracking Alzheimer's disease.

Usefulness - When asked about the usefulness of the system, all the caregivers were unanimous to the fact that it would be a great help on their job and on keeping them in a more regular contact with the medical staff responsible for their patient's treatment.

TABLE II. SUBJECTS' RESULTS WHEN PERFORMING THE USABILITY TESTS

\begin{tabular}{|c|c|c|c|c|}
\hline Subject & $\begin{array}{c}\text { Answer } \\
\text { interview? }\end{array}$ & $\begin{array}{c}\text { Consult } \\
\text { FAQ? }\end{array}$ & $\begin{array}{c}\text { Send } \\
\text { occurrence? }\end{array}$ & $\begin{array}{c}\text { Nr. of } \\
\text { errors }\end{array}$ \\
\hline 1 & Yes & Yes & Yes & 0 \\
\hline 2 & Yes & Yes & Yes & 2 \\
\hline 3 & Yes & Yes & Yes & 4 \\
\hline 4 & Yes & Yes & Yes & 0 \\
\hline 5 & Yes & Yes & Yes & 0 \\
\hline 6 & Yes & Yes & Yes & 2 \\
\hline 7 & Yes & Yes & Yes & 0 \\
\hline 8 & Yes & Yes & Yes & 1 \\
\hline 9 & Yes & Yes & Yes & 0 \\
\hline 10 & Yes & Yes & Yes & 0 \\
\hline
\end{tabular}

\section{CONCLUSION AND FUTURE WORK}

Being an incurable disease with symptoms that cause extreme burden in patients and caregivers, new ways of diagnosing, tracking and rehabilitating patients with $\mathrm{AD}$ are urgent.

While STAlz is still in an initial phase, its results are quite promising, suggesting that the use of technology to support the diagnosis, tracking and rehabilitation of Alzheimer's disease patients, can provide new courses of treatment, improvements in cognitive rehabilitation (or at least preventing deterioration) and allowing the disease to be better handled not only by the medical professionals but also by the caregiver and the patient itself.
A phase of testing with $\mathrm{AD}$ patients is already underway, although without any presentable results at this point. The future work will be focused on testing the system in a live environment (patient and caregiver at home without supervision) and adjusting the system based on the feedback gathered, and on testing the web platform with medical professional to adjust the metrics gathered and usability.

\section{ACKNOWLEDGMENT}

The authors would like to thank the caregivers that voluntarily participated in the usability tests and Dr. João Massano and everyone in his team of medical professionals for their overall insight and availability.

\section{REFERENCES}

[1] I. Plaza, L. Martín, S. Martin, and C. Medrano, "Mobile applications in an aging society: Status and trends," Journal of Systems and Software, vol. 84, no. 11, pp. 1977-1988, Nov. 2011. [Online]. Available: http://www.sciencedirect.com/science/article/pii/S016412121100135X

[2] N. Armstrong, C. D. Nugent, G. Moore, and D. D. Finlay, "Developing smartphone applications for people with Alzheimer's disease," pp. 1-5, 2010.

[3] A. Milne, A. Culverwell, R. Guss, J. Tuppen, and R. Whelton, "Screening for dementia in primary care: a review of the use, efficacy and quality of measures," International Psychogeriatrics, vol. 20, no. 05, pp. 911-926, 2008.

[4] A. Association, "National Alliance for Caregiving.(2004)," Families care: Alzheimer's caregiving in the United States, 2008. [Online]. Available: http://www.alz.org/national/documents/report_familiescare.pdf

[5] G. Cipriani, A. Bianchetti, and M. Trabucchi, "Outcomes of a computer-based cognitive rehabilitation program on Alzheimer's disease patients compared with those on patients affected by mild cognitive impairment," Archives of Gerontology and Geriatrics, vol. 43, no. 3, pp. 327-335, Nov. 2006. [Online]. Available: http://www.sciencedirect.com/science/article/pii/S0167494306000045

[6] L. D. Vreese and M. Neri, "Memory rehabilitation in Alzheimer's disease: a review of progress," ... journal of geriatric ..., no. December 2000, pp. 794-809, 2001. [Online]. Available: http://onlinelibrary.wiley.com/doi/10.1002/gps.428/abstract

[7] R. Ávila, "Resultados da reabilitação neuropsicológica em paciente com doença de Alzheimer leve," vol. 30, no. 4, pp. 139-146, 2003.

[8] C. M. C. Bottino, I. A. M. Carvalho, A. M. M. A. Alvarez, R. Avila, P. R. Zukauskas, S. E. Z. Bustamante, F. C. Andrade, S. R. Hototian, F. Saffi, and C. H. P. Camargo, "DOENÇA DE ALZHEIMER Relato de trabalho em equipe multidisciplinar," vol. 60, no. 99, pp. 70-79, 2002.

[9] CogWeb, "Treino cognitivo online validade cientificamente," Available at http://www.cogweb.pt, 2012.

[10] Lumosity, "Your brain, just brigther," Available at http://www.lumosity.com, 2012.

[11] RehaCom, "Cognitive rehabilitation with computers," Available at http://www.hasomed.de/en/products/rehacom-cognitive-therapy.html, 2012.

[12] S. H. Zarit, K. E. Reever, and J. Bach-Peterson, "Relatives of the impaired elderly: Correlates of feelings of burden," The Gerontologist, vol. 20, no. 6, pp. 649-655, 1980. [Online]. Available: http://gerontologist.oxfordjournals.org/content/20/6/649.abstract

[13] M. P. Lawton and E. M. Brody, "Assessment of older people: Selfmaintaining and instrumental activities of daily living," The Gerontologist, vol. 9, no. 3 Part 1, pp. 179-186, 1969. [Online]. Available: http://gerontologist.oxfordjournals.org/content/9/3_Part_1/179.short

[14] D. I. Kaufer, J. L. Cummings, P. Ketchel, V. Smith, A. MacMillan, T. Shelley, O. L. Lopez, and S. T. DeKosky, "Validation of the npi-q, a brief clinical form of the neuropsychiatric inventory," The Journal of neuropsychiatry and clinical neurosciences, vol. 12, no. 2, pp. 233-239, 2000. 